\title{
Lifestyle modifications for patients with breast cancer to improve prognosis and optimize overall health
}

\author{
Julia Hamer HBSc, Ellen Warner MD MSc
}

Cite as: CMAJ 2017 February 21;189:E268-74. doi: 10.1503/cmaj.160464

CMAJ Podcasts: author interview at https://soundcloud.com/cmajpodcasts/160464-view

A Ithough more than $90 \%$ of patients with breast cancer have early stage disease at diagnosis, about $25 \%$ will eventually die of distant metastasis. ${ }^{1}$ Many patients with breast cancer seek information from a variety of sources about behaviours that may reduce their risk of recurrence. ${ }^{2}$ Making positive lifestyle changes can also be psychologically beneficial to patients by empowering them, since the feeling of loss of control is one of biggest challenges of a cancer diagnosis.

In this review, we identify which lifestyle changes can be recommended to patients as an adjunct to standard breast cancer treatments, to reduce their risk of distant recurrence and death. We review the role of lifestyle factors, particularly weight management, exercise, diet, smoking, alcohol intake and vitamin supplementation, on the prognosis of patients with breast cancer. Our literature search is summarized in Box 1.

It is challenging to study lifestyle factors independently, because patients who are more physically active often are leaner, eat a healthier diet and are typically less likely to drink excessive amounts of alcohol or smoke; however, many studies try to adjust for these confounders. ${ }^{3}$ Another limitation is the potential impact of lifestyle factors before diagnosis. ${ }^{4}$ Regrettably, but understandably, we found very few randomized trials.

\section{How does body weight influence the prognosis for breast cancer?}

Women who gain weight during or after treatment of breast cancer have been consistently shown to be at higher risk of breast cancer-related death..$^{5-7}$ Also, women who are overweight or obese at the time of diagnosis have a poorer prognosis. ${ }^{8}$ Proposed mechanisms of how obesity might affect breast cancer mortality include a rise in circulating insulin-like growth factor, elevated circulating sex hormones and production of proinflammatory cytokines. ${ }^{9-12}$ Another possible mechanism is metabolic syndrome (presence of at least three of the following five components: abdominal obesity, hypertension, low level of highdensity lipoproteins, high plasma glucose level and high triglyceride levels). ${ }^{13}$ In a recent study involving early stage breast cancer survivors, patients with metabolic syndrome were at sig-

\section{KEY POINTS}

- Physical activity has the strongest effect on reducing the risk of breast cancer recurrence and death.

- All patients with breast cancer (except for those who have an abnormally low body mass index before diagnosis) should avoid gaining weight.

- Patients who are obese and those who smoke have a higher risk of cancer recurrence, but it is unclear whether their prognosis can be improved by weight loss or smoking cessation.

- Soy consumption is not harmful.

nificantly increased risk of distant metastasis (hazard ratio [HR] 2.45, 95\% confidence interval [Cl] 1.24-4.82) compared with those without the syndrome. ${ }^{13}$

Most patients with breast cancer gain weight both during and after active treatment, and much of the weight is never lost. The average reported weight gain is $2.5-5 \mathrm{~kg}$, ${ }^{5}$ but a gain of $10 \mathrm{~kg}$ or more is not uncommon. ${ }^{14}$ The reasons for weight gain are multifactorial and include "stress eating," reduced activity because of fatigue or other treatment-related adverse effects, lowered metabolic rate from chemotherapy, and use of pre- and post-chemotherapy medications such as dexamethasone. ${ }^{14}$ Weight gain is most common in women who experience treatment-related menopause and is often accompanied by relative fat gain and muscle loss. ${ }^{15}$

Several observational studies have shown that gaining weight during or after breast cancer treatment increases the risk of recurrence and reduces survival, irrespective of baseline body mass index (BMI). ${ }^{5-7}$ In a recent meta-analysis of 12 studies on weight gain after diagnosis, moderate weight gain (5\%-10\% of baseline weight) was not associated with increased mortality, but a weight gain of more than $10 \%$ was ( $\mathrm{HR} 1.17,95 \% \mathrm{Cl} 1.00-1.38$ ). There was no effect on breast cancer mortality with a weight gain of more than $5 \%$ among women with a pre-diagnostic BMI of 25 or higher. ${ }^{16}$

Because being overweight is a risk factor for breast cancer among postmenopausal women, a disproportionate number of women with breast cancer are above their ideal body weight at the time of diagnosis. Over the last five decades, many studies 


\section{Box 1: Evidence used in this review}

We searched PubMed to identify relevant systematic reviews and meta-analyses published within the past 10 years. Key search terms used with "breast cancer recurrence," "breast cancer survival" and "breast cancer mortality" included "weight loss," "weight gain" and "weight management" (10 reviews); "exercise" and "physical activity" (4 reviews); "diet," "nutrition," "dietary fat" and "soy" (8 reviews); "smoking" (3 reviews); "alcohol" (2 reviews); and "vitamin supplementation," "vitamins," "vitamin C," "vitamin E," "vitamin D" and "multivitamins" (5 reviews). A total of 31 reviews or meta-analyses were included ( 1 article was used for two lifestyle factors). We added 36 primary research articles because they were published after the most recent review of that lifestyle factor, they were designated in one of the reviews as a landmark study, or there were no previous reviews for that lifestyle factor. Details of the selection of articles are shown in Appendix 1 (available at www.cmaj.ca/lookup/suppl/doi:10.1503/cmaj.160464/-/DC1).

have shown a higher risk of breast cancer recurrence and mortality among patients who were obese at the time of diagnosis. A meta-analysis of 79 published studies found that, compared with women with a normal weight, those who were obese (BMI > 30) or overweight (BMI 25-30) at diagnosis had significantly higher breast cancer mortality (obese: summary relative risk [RR] 1.35, 95\% Cl 1.24-1.47; overweight: RR 1.11, 95\% Cl 1.06-1.17). ${ }^{8}$ Allcause mortality was similarly increased. The summary RR for each $5-\mathrm{kg} / \mathrm{m}^{2}$ increase in baseline BMI was 1.18 (95\% Cl 1.121.25), but substantial heterogeneity was found among the studies. No significant differences were seen between pre- and postmenopausal women. However, in a patient-level meta-analysis of randomized adjuvant studies, increased breast cancer mortality was observed only in the subgroup of heavier premenopausal women with estrogen-receptor-positive breast cancer. ${ }^{17}$

One might then hypothesize that overweight patients who lose weight after a breast cancer diagnosis would have a better outcome. Although multiple studies have shown that weight loss, at least in the short term, is achievable in breast cancer survivors through a variety of strategies, ${ }^{9,18}$ there are few data on long-term outcomes. ${ }^{19}$ In the randomized Women's Intervention Nutrition Study, which will be discussed later, patients in the dietary intervention arm lost an average of six pounds $(2.7 \mathrm{~kg}$ ) over a five-year period and had a lower incidence of disease recurrence (HR 0.76, $95 \% \mathrm{Cl} 0.60-0.98$ ) compared with the control group. ${ }^{20}$ However, this difference may have been attributable to the $15 \%$ reduction in dietary fat intake in the intervention group.

There is not yet a clear answer regarding the effect of weight loss, or prevention of weight gain, on the prognosis of patients with breast cancer. Two large randomized studies are underway to evaluate the promotion of weight management on breast cancer outcomes. ${ }^{19,21}$

\section{What role can exercise play in improving prognosis?}

A recent review of the effect of lifestyle factors on breast cancer mortality concluded that physical activity has the most robust effect of all lifestyle factors on reducing breast cancer recurrence. ${ }^{22}$
Lowered endogenous hormone levels, reduction of inflammation and reversal of insulin resistance have all been hypothesized to mediate the effects of exercise. ${ }^{23}$

A meta-analysis of 22 prospective cohort studies found that breast cancer mortality was significantly reduced among women who reported participating in recreational physical activity after their breast cancer diagnosis (HR 0.59, 95\% Cl 0.45-0.78). However, there was considerable heterogeneity across studies. The effect was stronger among women who met recommended levels of physical activity (see below), postmenopausal women and women with a BMI greater than 25 . In most studies, the effect was independent of the level of activity before the cancer diagnosis. ${ }^{23}$ These studies were controlled for baseline BMI; however, weight loss and weight gain were rarely monitored.

Patients should be encouraged to engage in at least 30 minutes of moderate-intensity physical activity at least five days of the week, or 75 minutes of more vigorous exercise, along with two to three weekly strength training sessions, including exercises for major muscle groups. This recommendation has been endorsed by both the Canadian Cancer Society ${ }^{24}$ and the American Cancer Society. ${ }^{25}$ However, more hours of exercise (but not more vigorous activity) may have increased benefit. Two analyses showed a substantial inverse dose-response effect between hours per week engaged in physical activity and breast cancer mortality. ${ }^{26,27}$ Similar beneficial metabolic effects have been shown for both aerobic and resistance exercise, but optimal results are achieved with a combination of the two. ${ }^{22}$

A population-based study in the United States involving 856 women found that only $13 \%$ of breast cancer survivors attained the recommended 150 minutes of exercise each week, and with increasing time after diagnosis, only about $10 \%$ complied with the recommendation..$^{28}$ Patients who have undergone chemotherapy or radiation have significantly greater decreases in physical activity, by $50 \%$ and $24 \%$ respectively, compared with patients who have not experienced these therapies. ${ }^{22}$

\section{Can a change in diet improve outcomes?}

\section{Fats}

Preclinical research suggests that excess dietary intake of lipids ${ }^{29}$ and carbohydrates can influence metabolic and hormonal processes (e.g., by increasing endogenous estrogen levels) that affect the development of breast cancer metastasis. ${ }^{30,31}$ Clinical research is hindered by the variability in diets and the associated metabolic interactions that may occur when trying to study the effects of specific macronutrients. Weight and physical activity can also confound results of dietary studies.

Several observational studies have suggested that high consumption of saturated fats ${ }^{32}$ and high-fat dairy products may be associated with an increased risk of breast cancer mortality. ${ }^{3,33}$ One study involving 1893 women showed that high intake of high-fat dairy products (> 1 serving daily) was significantly more detrimental than an intake of less than 0.5 servings daily (HR $1.49,95 \% \mathrm{Cl} 1.00-2.24)$. Low-fat dairy products were not associated with an increased risk of death related to breast cancer. ${ }^{3}$ However, BMI and the amount of physical activity may have con- 
founded the results of these dietary studies and should be controlled for in future interventional trials. ${ }^{33}$

In the Women's Intervention Nutrition study, in which more than 2400 postmenopausal women with breast cancer were randomly assigned to a dietary intervention (five-year intervention to reduce their dietary fat intake by $15 \%$ ) or to a control group, the incidence of recurrence was significantly lower in the intervention group (HR 0.76, 95\% Cl 0.60-0.98)..$^{20}$ As previously mentioned, it cannot be determined whether this difference was due to the sixpound average weight loss or the reduction in dietary fat intake in the intervention group.

\section{Specific diets}

There is currently no particular style of diet that has been found to be more beneficial than another for reducing the risk of breast cancer recurrence. ${ }^{34}$ Studies comparing a "prudent" diet (high in fruits, vegetables, whole grains and chicken) to a Western-style diet (high in processed grains, processed meats and red meat) have shown equivalent rates of recurrence. ${ }^{4,35}$ In the prospective Women's Health Initiative's Dietary Modification Trial, postmenopausal women with breast cancer who consumed better quality diets (as measured by the validated Healthy Eating Index-2005 calculated from a food frequency questionnaire that was completed an average of 1.5 years after breast cancer diagnosis) did not have a reduced risk of death from breast cancer (HR 0.91, 95\% Cl 0.60-1.40). ${ }^{36}$

Although a recent meta-analysis did not find a significant effect from a Mediterranean diet (a diet rich in vegetables, unsaturated fats, fruits, fish and whole grains, with moderate red wine intake and limited intake of red meat and simple carbohydrates) on cancer recurrence, ${ }^{37}$ several other studies found that a diet similar to the Mediterranean diet (but without the specification to include red wine) had a beneficial effect on breast cancer survival. ${ }^{37,38}$ The ongoing prospective DIANA (Diet and Androgens)-5 trial aims to determine whether a Mediterranean diet combined with regular physical activity can improve breast cancer outcomes. ${ }^{39}$

\section{Soy}

Many patients are advised by health care professionals, or find information on the Internet, to avoid soy because it contains estrogens. However, findings from clinical studies do not support this recommendation. Soybeans contain both soy isoflavones, which are phytoestrogens, and soy proteins, which can be isolated and extracted. Depending on the extraction process, varying amounts of soy isoflavones can be found in soy protein. ${ }^{40}$

Soy protein isolates increase insulin-like growth factor 1 when consumed in high amounts, which could theoretically promote breast cancer recurrence. ${ }^{41}$ One clinical study found that soy protein isolate supplementation caused a slight stimulatory effect on epithelial breast cells in premenopausal women. ${ }^{42}$

Soy products high in isoflavones, such as edamame, tofu, tempeh and miso soup, have been found to have anticarcinogenic effects in preclinical studies, having been associated with apoptosis, antiangiogenesis and reduced sex hormone levels. ${ }^{41}$ One study found that supplementation with $200 \mathrm{mg}$ of soy isoflavones daily over two to six weeks before breast cancer surgery showed a non- significant trend toward inhibition of cancer growth compared with a control group. ${ }^{43}$

A meta-analysis of five prospective cohort studies from the United States and China found that high consumption of soy protein or soy isoflavones after breast cancer diagnosis was associated with a $26 \%$ decrease in recurrence ( $\mathrm{HR} 0.74,95 \% \mathrm{Cl} 0.61-0.85$ ) and a $16 \%$ reduction in breast cancer mortality (HR $0.84,95 \% \mathrm{Cl}$ $0.71-0.99)$ compared with low consumption. ${ }^{44}$ Another meta-analysis of four prospective cohort studies, two of which were included in the previous analysis, found a $16 \%$ reduced risk of recurrence among women consuming high levels of soy isoflavone (RR 0.84, $95 \% \mathrm{Cl}$ 0.65-0.86), with no significant heterogeneity among the studies. ${ }^{45}$ Interestingly, in both meta-analyses, soy intake was not associated with improved survival or reduced recurrence among premenopausal women or patients who used tamoxifen, which suggests that soy and tamoxifen may work through a similar mechanism of displacing estrogen from its receptor. ${ }^{44}$ In some studies, the apparent benefit of soy was confined to patients with estrogen-receptor-positive tumours. Phytoestrogens bind to some estrogen receptors to mimic estrogen, whereas others antagonize and block the receptors; however, the selectivity of soy isoflavones is still not well understood. ${ }^{46}$

Although randomized trials would be necessary to confirm that soy intake truly reduces breast cancer recurrence, there is sufficient evidence to at least conclude that soy products need not be avoided. Regardless of whether soy independently reduces recurrence, increasing dietary soy intake may help facilitate weight management if soy products replace higher-calorie sources of protein such as meat.

\section{Is there a benefit from quitting smoking and reducing alcohol consumption?}

Recent observational studies have shown that women with breast cancer who have a substantial smoking history have increased breast cancer mortality compared with those who never smoked. ${ }^{47,48}$ It is still uncertain whether quitting smoking after a breast cancer diagnosis affects breast cancer recurrence.

In a recent prospective observational study involving 20691 women with breast cancer, those who continued to smoke after diagnosis ( $10 \%$ of the study population) were more likely than those who never smoked to die of breast cancer (HR 1.72, 95\% Cl 1.13-2.60), ${ }^{48}$ a finding that has been observed by others. ${ }^{49-52} \mathrm{Com}$ pared with women who continued to smoke after diagnosis, those who quit had lower rates of death from breast cancer (HR 0.67, 95\% $\mathrm{Cl} 0.38-1.19)$ and respiratory cancer (HR 0.39, 95\% Cl 0.16-0.95), but only the latter was statistically significant. ${ }^{48}$ The study was well controlled for cancer stage and multiple prognostic factors.

The evidence is not strong enough to overturn the 2014 conclusion of the US Surgeon General that there is insufficient evidence to recommend smoking cessation to reduce breast cancer recurrence. ${ }^{47}$ However, there are still several reasons to strongly encourage patients with recently diagnosed breast cancer to quit smoking. These include increased overall mortality among breast cancer survivors who are smokers ${ }^{53}$ and an increased risk of venous thrombosis among smokers taking tamoxifen. ${ }^{54}$ 
Studies of alcohol consumption after a breast cancer diagnosis have not shown a consistent association with disease recurrence. $\mathrm{A}$ recent meta-analysis of 14 cohort studies concluded that postdiagnosis alcohol consumption was not associated with breast cancer mortality, although subgroup analyses found that high intake of alcohol (> $20 \mathrm{~g} / \mathrm{d}$ ) was associated with higher breast cancer mortality, with a dose-response association among premenopausal women. ${ }^{55}$ A subsequent pooled analysis of four cohort studies reported that alcohol intake of more than one drink daily was associated with a $28 \%$ increased risk of late recurrence (> $5 \mathrm{yr}$ after diagnosis) $(95 \% \mathrm{Cl}$ 1.01-1.62) among survivors with estrogen-receptor-positive breast cancer..$^{50}$ Overall, the results are inconclusive. Nevertheless, limiting alcohol consumption to one or fewer drinks per day is a worthwhile goal to reduce the risk of a second primary breast cancer. ${ }^{54}$

\section{Is vitamin supplementation helpful?}

\section{Vitamins $\mathrm{C}$ and $\mathrm{E}$}

Patients are often told to avoid taking supplements of antioxidant vitamins such as $\mathrm{C}$ and $\mathrm{E}$ during chemotherapy and radiation because of concerns that these supplements may negate the treatment effects that are mediated, at least in part, by free-radical formation. However, a recent meta-analysis of 10 prospective observational studies of supplemental and dietary vitamin $C$ intake after breast cancer diagnosis ( $<400 \mathrm{mg} / \mathrm{d}$ in most studies and no more than $1000 \mathrm{mg}$ ) found a $15 \%$ reduction in breast cancer mortality in the supplement group (summary RR 0.85 , 95\% Cl 0.740.99 ) and a $22 \%$ reduction for a $100-\mathrm{mg} / \mathrm{d}$ increase in dietary vitamin C (summary RR $0.78,95 \% \mathrm{Cl} 0.64-0.94) .{ }^{56}$ These studies did not correct for BMI, use of other supplements, other dietary components or physical activity. There was no clear detrimental effect of vitamin $\mathrm{C}$ taken during chemotherapy or radiation. Randomized trials to confirm these findings are warranted.

Most studies have not found an association between vitamin $\mathrm{E}$ supplementation and reduced breast cancer recurrence or breast cancer mortality. ${ }^{57,58}$

\section{Vitamin D}

Low levels of 25-hydroxyvitamin D may be associated with an increased risk of death from breast cancer. ${ }^{59,60}$ Vitamin D exhibits pro-differentiation and anti-proliferation properties. ${ }^{12}$

A meta-analysis of six prospective studies, including cohort and nested case-control studies, found that higher 25-hydroxyvitamin $D$ levels were associated with a lower risk of breast cancer mortality (pooled RR $0.58,95 \% \mathrm{Cl} 0.40-0.85$ ), with no evidence of heterogeneity. ${ }^{61}$ Each $10-\mathrm{ng} / \mathrm{mL}(25-\mathrm{nmol} / \mathrm{L})$ increment in blood 25 -hydroxyvitamin $\mathrm{D}$ levels was associated with a 0.88 relative risk in breast cancer mortality $(95 \% \mathrm{Cl} 0.79-0.98)$ and a 0.84 relative risk in overall mortality $(95 \% \mathrm{Cl} 0.78-0.91)$. The association with supplemental vitamin D was only slightly stronger than dietary intake of vitamin D.

Another meta-analysis of five studies showed that higher serum 25-hydroxyvitamin D levels at diagnosis were associated with lower breast cancer mortality. ${ }^{62}$ Patients with vitamin D levels in the highest quintile had about half the death rate of those with levels in the lowest quintile (HR 0.56, 95\% Cl 0.40-0.70). Two earlier studies showed that higher vitamin $D$ intake was associated with a lower risk of recurrence among premenopausal patients $(p=0.02)^{63}$ and among patients with estrogen-receptor-positive tumours $(p=0.01) .{ }^{58}$ Moreover, a retrospective study involving patients with HER2-positive breast cancer showed significant improvement in disease-free survival among those given trastuzumab and a vitamin $\mathrm{D}$ supplement $(<1500 \mathrm{IU} / \mathrm{d})(\mathrm{HR} 0.36,95 \% \mathrm{Cl} 0.15-0.88)$ compared with patients receiving the same treatment without a vitamin D supplement. ${ }^{64}$

Monitoring serum 25-hydroxyvitamin D levels in breast cancer patients may be prudent, with a goal of maintaining a concentration within the normal range of $30-80 \mathrm{ng} / \mathrm{mL}(75-200 \mathrm{nmol} / \mathrm{L}){ }^{62}$ Although randomized clinical trials are warranted to confirm the effect of vitamin D supplementation on breast cancer survival, there are other reasons to optimize patients' vitamin D levels. Chemotherapy, tamoxifen and ovarian suppression all reduce the bone density of premenopausal women, and aromatase inhibitors lower bone density and increase the risk of fractures, particularly in postmenopausal women.

\section{Multivitamins}

A review of four cohort studies on multivitamin supplementation after breast cancer diagnosis found no association with breast cancer recurrence or breast cancer mortality. However, the subgroup of patients with estrogen-receptor-negative disease had a $25 \%$ reduced risk of recurrence with multivitamin supplementation after diagnosis (HR 0.75, 95\% Cl 0.59-0.95)..$^{58}$

\section{Which lifestyle modifications should be encouraged?}

A summary of key findings is presented in Box 2 .

Of all lifestyle factors, physical activity has the most robust effect on breast cancer outcomes. Following the recommended 150 minutes of moderate to vigorous exercise or 75 minutes of vigorous exercise per week, along with two to three weekly sessions of strength training, can help reduce the risk of breast cancer recurrence and mortality. Because it is common for patients to reduce their level of physical activity after a breast cancer diagnosis, it is important for health care professionals to promote and encourage exercise in this patient population. Simply receiving advice from an oncologist to exercise more has been shown to increase patients' level of physical activity ${ }^{65}$ Although cancer centres are increasingly developing short-term exercise programs for their patients, primary care providers have an essential role in reinforcing the need to turn exercise into a life-long habit. Some experts have recommended having systems in place to refer patients directly from a cancer care clinic to community-based exercise programs specialized for cancer survivors. ${ }^{66}$ Although specific exercise interventions can result in strong behavioural changes, providing support to patients by phone or email has also proven effective. ${ }^{67}$

Weight gain of more than $10 \%$ body weight after a breast cancer diagnosis increases breast cancer mortality and all-cause mortality. However, there are good reasons to discourage even moderate weight gain because of its negative effects on mood and body image. ${ }^{68}$ Again, physical activity is key. Women who participate in an aerobic exercise program while undergoing adjuvant chemo- 
therapy generally do not gain weight, whereas those who do not exercise gain $3.2 \mathrm{~kg}$ on average. ${ }^{5}$ The benefits of exercise extend well beyond effects on prognosis, including substantial selfperceived improvements in appearance, cardiorespiratory fitness, lymphedema and emotional well-being. ${ }^{22}$

Obesity at diagnosis has a negative impact on breast cancer prognosis, but it is still unclear whether weight loss improves the prognosis of overweight and obese women.

Patients should be strongly encouraged to quit smoking to improve overall survival and possibly breast cancer-specific sur-

\section{Box 2: Summary of key findings}

\section{Weight management}

- Weight gain during or after breast cancer treatment increases the risk of recurrence and reduces survival, irrespective of baseline body mass index. ${ }^{5-7}$

- Patients who are obese or overweight at breast cancer diagnosis have a poorer prognosis. ${ }^{8}$ Although it is possible for such patients to attain meaningful weight loss, ${ }^{9,18}$ there are few data on whether this improves breast cancer outcomes. ${ }^{19}$

\section{Physical activity}

- Physical activity can reduce breast cancer mortality by about $40 \%{ }^{23}$ and has the most powerful effect of any lifestyle factor on breast cancer outcomes. ${ }^{22}$

- At least 150 minutes per week of physical activity is recommended, ${ }^{24,25}$ but less than $13 \%$ of patients with breast cancer attain this. ${ }^{28}$

\section{Diet}

- Western-style diets (high in processed grains, processed meats and red meat) and prudent diets (high in fruits, vegetables, whole grains and chicken) have similar rates of breast cancer recurrence. ${ }^{4,35}$

- Dietary saturated fat, especially from high-fat dairy products, may be associated with increased breast cancer mortality. ${ }^{3,32,33}$

- Soy products have not been found to increase breast cancer recurrence ${ }^{41}$ and may actually reduce it. ${ }^{44,45}$

\section{Smoking}

- Recent evidence has shown a strong association between a history of smoking and breast cancer mortality. ${ }^{47}$

- Compared with women who continue to smoke after a breast cancer diagnosis, those who quit smoking after diagnosis have higher overall survival and possibly better breast cancer-specific survival. ${ }^{48-52}$

\section{Alcohol intake}

- Findings are too inconsistent to conclude that alcohol consumption affects breast cancer outcomes. However, limiting alcohol consumption to one or fewer drinks per day reduces the risk of a second primary breast cancer. ${ }^{54}$

\section{Vitamin supplementation}

- Moderate increases in dietary vitamin C or oral supplementation may reduce breast cancer mortality, ${ }^{56}$ but randomized trials are needed to confirm these findings.

- Vitamin E supplementation is not associated with breast cancer outcomes. ${ }^{57,58}$

- Low levels of serum 25-hydroxyvitamin D at diagnosis have been associated with an increased risk of breast cancer mortality. ${ }^{59-62}$ However, randomized trials are needed to determine whether supplementation improves prognosis. vival. ${ }^{47}$ Presenting evidence to patients who smoke that it's not too late to benefit from quitting, ${ }^{47,69}$ carefully monitoring patients' smoking status at each visit, and recommending pharmacologic or behavioural therapy ${ }^{70}$ are all strategies that can aid patients with smoking cessation.

There is no evidence to support a recommendation that patients with breast cancer refrain from eating soy products; increasing intake of soy products may be protective.

Randomized trials are needed to confirm whether decreased intake of saturated fat (especially dairy fat), increased soy consumption, moderation of alcohol intake, and increased vitamin C or $\mathrm{D}$ supplementation can reduce breast cancer mortality (Box 3 ). Results of ongoing interventional studies are eagerly awaited. In the meantime, most patients would benefit from vitamin $D$ supplementation, at least to optimize bone health.

An important point to emphasize is that lifestyle changes, although often protective, cannot always improve outcomes of cancer with particularly aggressive biology. Patients should not be made to feel that inadequate lifestyle changes have led to recurrence of their cancer.

Although most patients with breast cancer die from other causes, a cancer diagnosis creates a "teachable moment" when patients are more receptive to healthy lifestyle changes. Regardless of whether these changes affect the prognosis, they will almost certainly improve patients' overall health.

\section{Box 3: Unanswered questions}

- Does weight loss after diagnosis improve survival for obese/overweight patients?

- Can a reduction in dietary saturated fat or high-fat dairy products improve prognosis?

- Are soy isoflavones protective against recurrence?

- Does quitting smoking after a breast cancer diagnosis decrease breast cancer mortality?

- What should the weekly limit of alcohol consumption be for patients with breast cancer?

- Does vitamin C or D supplementation reduce the risk of distant recurrence?

\section{References}

1. O'Shaughnessy J. Extending survival with chemotherapy in metastatic breast cancer. Oncologist 2005;10(Suppl 3):20-9.

2. Kwok A, Palermo C, Boltong A. Dietary experiences and support needs of women who gain weight following chemotherapy for breast cancer. Support Care Cancer 2015;23:1561-8.

3. Kroenke CH, Kwan ML, Sweeney C, et al. High- and low-fat dairy intake, recurrence, and mortality after breast cancer diagnosis. J Natl Cancer Inst 2013; 105:616-23.

4. Kroenke $\mathrm{CH}$, Fung TT, Hu FB, et al. Dietary patterns and survival after breast cancer diagnosis. J Clin Oncol 2005;23:9295-303.

5. Ghose A, Kundu R, Toumeh A, et al. A review of obesity, insulin resistance, and the role of exercise in breast cancer patients. Nutr Cancer 2015;67:197-202.

6. Caan BJ, Kwan ML, Shu XO, et al. Weight change and survival after breast cancer in the After Breast Cancer Pooling Project. Cancer Epidemiol Biomarkers Prev 2012;21:1260-71.

7. Bradshaw PT, Ibrahim JG, Stevens J, et al. Postdiagnosis change in bodyweight and survival after breast cancer diagnosis. Epidemiology 2012;23:320-7. 
8. Chan DS, Vieira AR, Aune D, et al. Body mass index and survival in women with breast cancer - systematic literature review and meta-analysis of 82 follow-up studies. Ann Oncol 2014;25:1901-14.

9. Playdon M, Thomas $G$, Sanft $T$, et al. Weight loss intervention for breast cancer survivors: a systematic review. Curr Breast Cancer Rep 2013;5:222-46.

10. Rodríguez San Felipe MJ, Aguilar Martinez A, Manuel-y-Keenoy B. Influence of body weight on the prognosis of breast cancer survivors; nutritional approach after diagnosis. Nutr Hosp 2013;28:1829-41.

11. Goodwin PJ. Obesity, insulin resistance and breast cancer outcomes. Breast 2015; 24(Suppl 2):S56-9.

12. Brenner DR, Brockton NT, Kotsopoulos J, et al. Breast cancer survival among young women: a review of the role of modifiable lifestyle factors. Cancer Causes Control 2016;27:459-72.

13. Berrino F, Villarini A, Traina A, et al. Metabolic syndrome and breast cancer prognosis. Breast Cancer Res Treat 2014;147:159-65.

14. Irwin ML, Fabian C, McTiernan A. Risk reduction from weight management and physical activity interventions. Adv Exp Med Biol 2015;862:193-212.

15. Gadéa E, Thivat E, Planchat E, et al. Importance of metabolic changes induced by chemotherapy on prognosis of early-stage breast cancer patients: a review of potential mechanisms. Obes Rev 2012;13:368-80.

16. Playdon MC, Bracken MB, Sanft TB, et al. Weight gain after breast cancer diagnosis and all-cause mortality: systematic review and meta-analysis. J Natl Cancer Inst 2015;107:djv275.

17. Pan H, Gray RG; Early Breast Cancer Trialists' Collaborative Group; Clinical Trial Service Unit and Epidemiological Studies Unit, Nuffield Department of Population Health, University of Oxford, Oxford, United Kingdom; University of Oxford, Oxford, United Kingdom. Effect of obesity in premenopausal ER+ early breast cancer: EBCTCG data on 80000 patients in 70 trials [abstract 503]. J Clin Oncol 2014;32(Suppl 5).

18. Reeves MM, Terranova CO, Eakin EG, et al. Weight loss intervention trials in women with breast cancer: a systematic review. Obes Rev 2014;15:749-68.

19. Rock CL, Flatt SW, Byers TE, et al. Results of the Exercise and Nutrition to Enhance Recovery and Good Health for You (ENERGY) trial: a behavioral weight loss intervention in overweight or obese breast cancer survivors. J Clin Oncol 2015;33:3169-76.

20. Chlebowski RT, Blackburn GL, Thomson CA, et al. Dietary fat reduction and breast cancer outcome: interim efficacy results from the Women's Intervention Nutrition Study. J Natl Cancer Inst 2006;98:1767-76.

21. Rack B, Andergassen U, Neugebauer J, et al. The German SUCCESS C Study the first European lifestyle study on breast cancer. Breast Care (Basel) 2010;5: 395-400.

22. Dieli-Conwright CM, Orozco BZ. Exercise after breast cancer treatment: current perspectives. Breast Cancer (Dove Med Press) 2015;7:353-62.

23. Lahart IM, Metsios GS, Nevill AM, et al. Physical activity, risk of death and recurrence in breast cancer survivors: a systematic review and meta-analysis of epidemiological studies. Acta Oncol 2015;54:635-54.

24. Physical activity. Toronto: Canadian Cancer Society; 2016. Available: www. cancer.ca/en/prevention-and-screening/live-well/nutrition-and-fitness/physical -activity/?region=on (accessed 2016 June 24).

25. US Centers for Disease Control and Promotion. Promoting physical activity: a guide for community action. 2nd ed. Champaign (IL): Human Kinetics; 2010.

26. Beasley JM, Kwan ML, Chen WY, et al. Meeting the physical activity guidelines and survival after breast cancer: findings from the After Breast Cancer Pooling Project. Breast Cancer Res Treat 2012;131:637-43.

27. Chen X, Lu W, Zheng W, et al. Exercise after diagnosis of breast cancer in association with survival. Cancer Prev Res (Phila) 2011;4:1409-18.

28. Irwin ML, Crumley D, McTiernan A, et al. Physical activity levels before and after a diagnosis of breast carcinoma: the Health, Eating, Activity, and Lifestyle (HEAL) study. Cancer 2003;97:1746-57.

29. Kim J, Lee YH, Park JH, et al. Estrogen deprivation and excess energy supply accelerate 7,12-dimethylbenz(a)anthracene-induced mammary tumor growth in C3H/HeN mice. Nutr Res Pract 2015;9:628-36.

30. Jiang Y, Pan Y, Rhea PR, et al. A sucrose-enriched diet promotes tumorigenesis in mammary gland in part through the 12-lipoxygenase pathway. Cancer Res 2016;76:24-9.

31. Chlebowski RT, Aiello E, McTiernan A. Weight loss in breast cancer patient management. J Clin Oncol 2002;20:1128-43.

32. Makarem N, Chandran U, Bandera EV, et al. Dietary fat in breast cancer survival. Annu Rev Nutr 2013;33:319-48.

33. Patterson RE, Cadmus LA, Emond JA, et al. Physical activity, diet, adiposity and female breast cancer prognosis: a review of the epidemiologic literature. Maturitas 2010;66:5-15.

34. Villarini A, Pasanisi P, Raimondi M, et al. Preventing weight gain during adjuvant chemotherapy for breast cancer: a dietary intervention study. Breast Cancer Res Treat 2012;135:581-9.

35. Kwan ML, Weltzien E, Kushi LH, et al. Dietary patterns and breast cancer recurrence and survival among women with early-stage breast cancer. J Clin Oncol 2009;27:919-26.

36. George SM, Ballard-Barbash R, Shikany JM, et al. Better postdiagnosis diet quality is associated with reduced risk of death among postmenopausal women with invasive breast cancer in the Women's Health Initiative. Cancer Epidemiol Biomarkers Prev 2014;23:575-83.

37. Schwingshackl L, Hoffmann G. Adherence to Mediterranean diet and risk of cancer: an updated systematic review and meta-analysis of observational studies. Cancer Med 2015;4:1933-47.

38. Berrino F, Villarini A, De Petris M, et al. Adjuvant diet to improve hormonal and metabolic factors affecting breast cancer prognosis. Ann N Y Acad Sci 2006; 1089:110-8.

39. Villarini A, Pasanisi P, Traina A, et al. Lifestyle and breast cancer recurrences: the DIANA-5 trial. Tumori 2012;98:1-18.

40. Setchell KDR, Cole SJ. Variations in isoflavone levels in soy foods and soy protein isolates and issues related to isoflavone databases and food labeling. J Agric Food Chem 2003;51:4146-55.

41. Gonzales JF, Barnard ND, Jenkins DJ, et al. Applying the precautionary principle to nutrition and cancer. J Am Coll Nutr 2014;33:239-46.

42. Petrakis NL, Barnes S, King EB, et al. Stimulatory influence of soy protein isolate on breast secretion in pre- and postmenopausal women. Cancer Epidemiol Biomarkers Prev 1996;5:785-94.

43. Sartippour MR, Rao JY, Apple S, et al. A pilot clinical study of short-term isoflavone supplements in breast cancer patients. Nutr Cancer 2004;49:59-65.

44. Chi F, Wu R, Zeng YC, et al. Post-diagnosis soy food intake and breast cancer survival: a meta-analysis of cohort studies. Asian Pac J Cancer Prev 2013;14:2407-12.

45. Dong JY, Qin LQ. Soy isoflavones consumption and risk of breast cancer incidence or recurrence: a meta-analysis of prospective studies. Breast Cancer Res Treat 2011;125:315-23.

46. Wang LQ. Mammalian phytoestrogens: enterodiol and enterolactone. J Chromatogr B Analyt Technol Biomed Life Sci 2002;777:289-309.

47. Parker BA, Pierce JP. Importance of smoking cessation to reduce breast cancer mortality. J Clin Oncol 2016;34:1295-6.

48. Passarelli MN, Newcomb PA, Hampton JM, et al. Cigarette smoking before and after breast cancer diagnosis: mortality from breast cancer and smoking-related diseases. J Clin Oncol 2016;34:1315-22.

49. Pierce JP, Patterson RE, Senger CM, et al. Lifetime cigarette smoking and breast cancer prognosis in the After Breast Cancer Pooling Project. J Natl Cancer Inst 2014;106:djt359.

50. Nechuta S, Chen WY, Cai H, et al. A pooled analysis of post-diagnosis lifestyle factors in association with late estrogen-receptor positive breast cancer prognosis. Int J Cancer 2016;138:2088-97.

51. Carter BD, Abnet CC, Feskanich D, et al. Smoking and mortality-beyond established causes. NEngl J Med 2015;372:631-40.

52. Braithwaite D, Izano M, Moore DH, et al. Smoking and survival after breast cancer diagnosis: a prospective observational study and systematic review. Breast Cancer Res Treat 2012;136:521-33.

53. Eulenburg C, Schroeder J, Obi N, et al. A comprehensive multistate model analyzing associations of various risk factors with the course of breast cancer in a population-based cohort of breast cancer cases. Am J Epidemiol 2016;183: 325-34.

54. Goodwin PJ, Ambrosone CB, Hong CC. Modifiable lifestyle factors and breast cancer outcomes: current controversies and research recommendations. Adv Exp Med Biol 2015;862:177-92.

55. Gou YJ, Xie DX, Yang KH, et al. Alcohol consumption and breast cancer survival: a meta-analysis of cohort studies. Asian Pac J Cancer Prev 2013;14:4785-90.

56. Harris HR, Orsini N, Wolk A. Vitamin C and survival among women with breast cancer: a meta-analysis. Eur J Cancer 2014;50:1223-31.

57. Nechuta S, Lu W, Chen Z, et al. Vitamin supplement use during breast cancer treatment and survival: a prospective cohort study. Cancer Epidemiol Biomarkers Prev 2011;20:262-71.

58. Poole EM, Shu X, Caan BJ, et al. Postdiagnosis supplement use and breast cancer prognosis in the After Breast Cancer Pooling Project. Breast Cancer Res Treat 2013;139:529-37. 
59. Toriola AT, Nguyen N, Scheitler-Ring K, et al. Circulating 25-hydroxyvitamin D levels and prognosis among cancer patients: a systematic review. Cancer Epidemiol Biomarkers Prev 2014;23:917-33.

60. Jacobs ET, Kohler LN, Kunihiro AG, et al. Vitamin D and colorectal, breast, and prostate cancers: a review of the epidemiological evidence. J Cancer 2016; 7:232-40.

61. Kim Y, Je Y. Vitamin D intake, blood 25(OH)D levels, and breast cancer risk or mortality: a meta-analysis. Br J Cancer 2014;110:2772-84.

62. Mohr SB, Gorham ED, Kim J, et al. Meta-analysis of vitamin D sufficiency for improving survival of patients with breast cancer. Anticancer Res 2014;34:1163-6.

63. Jacobs ET, Thomson CA, Flatt SW, et al. Vitamin D and breast cancer recurrence in the Women's Healthy Eating and Living (WHEL) study. Am J Clin Nutr 2011;93:108-17.

64. Zeichner SB, Koru-Sengul T, Shah N, et al. Improved clinical outcomes associated with vitamin $\mathrm{D}$ supplementation during adjuvant chemotherapy in patients with HER2+ nonmetastatic breast cancer. Clin Breast Cancer 2015;15:e1-11.
65. Jones LW, Courneya KS, Fairey AS, et al. Effects of an oncologist's recommendation to exercise on self-reported exercise behavior in newly diagnosed breast cancer survivors: a single-blind, randomized controlled trial. Ann Behav Med 2004;28:105-13.

66. Schmitz KH. Exercise for secondary prevention of breast cancer: moving from evidence to changing clinical practice. Cancer Prev Res (Phila) 2011;4:476-80.

67. Bluethmann SM, Vernon SW, Gabriel KP, et al. Taking the next step: a systematic review and meta-analysis of physical activity and behavior change interventions in recent post-treatment breast cancer survivors. Breast Cancer Res Treat 2015; 149:331-42.

68. Befort CA, Austin H, Klemp JR. Weight control needs and experiences among rural breast cancer survivors. Psychooncology 2011;20:1069-75.

69. Fillon M. Continuing to smoke after breast cancer diagnosis lowers survival rate. J Natl Cancer Inst 2016;108:djw160.

70. Shields PG. New NCCN guidelines: smoking cessation for patients with cancer. $J$ Natl Compr Canc Netw 2015;13(Suppl 5):643-5.
Competing interests: None declared.

This article has been peer reviewed.

Affiliations: Division of Medical Oncology and Hematology, Odette Cancer Centre, Sunnybrook Health Sciences Centre, Toronto, Ont.
Contributors: Ellen Warner was responsible for the concept. Julia Hamer and Ellen Warner contributed to the acquisition, analysis and interpretation of data and drafted the work. Ellen Warner critically revised the manuscript. Both authors approved the final version to be published and agreed to act as guarantors of the work.

Correspondence to: Ellen Warner, ellen.warner@sunnybrook.ca 